\title{
A 20 años de las Primeras Jornadas Latinoamericanas de Género y Geografía. Entrevista a Mónica Colombara
}

\author{
Magdalena Moreno ${ }^{1}$ \\ Consejo Nacional de Investigaciones Científicas y Técnicas (CONICET) \\ Universidad Nacional del Centro de la Provincia de Buenos Aires (UNCPBA) \\ @ [ magdalenamorenoivan@gmail.com ]
}

DOI: http://dx.doi.org/10.19137/huellas-2018-2218

$\mathrm{L}$

a Geografía de Génedesarrollándose en tanto disciplina científica desde mediados de la década del '80 de manera sostenida. A partir de 1990 se consolidó su inicio con aportes que cuestionaban la producción científica de la disciplina por considerar que no incorporaba las desigualdades de género que existen en el espacio geográfico.

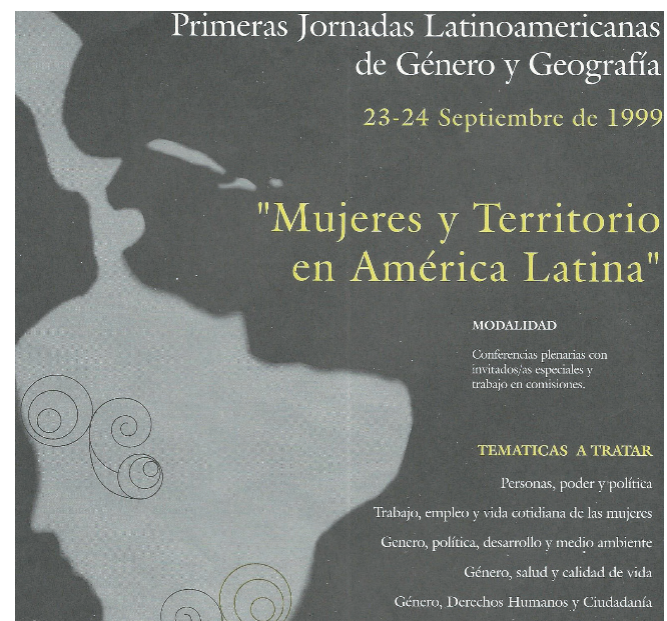
Como consecuencia del trabajo sostenido de las geógrafas que velaban por incorporar la perspectiva de género, en 1999 se produjeron dos hitos en la historia de la Geo-

1 Doctoranda en Geografía (UBA) sobre "La espacialidad de las redes de trata con fines de explotación sexual en tiempos del Bicentenario". Becaria doctoral de CONICET en el proyecto "Configuración y reconfiguración socioterritorial de la Argentina en tiempos del bicentenario." de la Unidad Ejecutora del Instituto de Geografía, Historia y Ciencias Sociales (Universidad Nacional del Centro de la Provincia de Buenos Aires). 
grafía de Género de Argentina: la publicación de un número especial de la Revista Huellas de la Universidad Nacional de La Pampa (UNLPam) y las Primeras Jornadas Latinoamericanas de Género y Geografía (en adelante, Jornadas) en la Universidad de Lomas de Zamora (UNLZ). En el presente trabajo se propone realizar una breve contribución a la recuperación de la historia de la Geografía de Género en Argentina, a través de una entrevista a la organizadora de las Jornadas.

El número especial de la Revista Huellas constó de cuatro partes: una presentación sobre la serie de publicaciones especiales realizada por la revista acerca diferentes perspectivas de la Geografía; un artículo específico sobre la temática realizado por Beatriz Dillon, que por entonces era la Directora del Departamento de Geografía de la UNLPam, denominado " $\mathrm{La}$ mitad del cielo'. Una introducción a la geografía del género.”; un comentario de María Di Liscia, que se desempeñaba como Directora del Instituto Interdisciplinario de Estudios de la Mujer de la UNLPam; y tres artículos que habían sido presentados en las V Jornadas de Historia de las Mujeres y Estudios de Género de $1998^{2}$.

Las Jornadas de la UNLZ estuvieron organizadas por la Lic. Mónica Colombara, pionera en introducir la perspectiva de Género en los estudios geográficos argentinos, y fueron auspiciadas por la Comisión de Geografía y Género de la Unión Geográfica Internacional (UGI). Las Jornadas se realizaron los días 23 y 24 de septiembre de 1999 y llevaron por lema "Mujeres y Territorio en América Latina", con diferentes temáticas a tratar. Se presentaron dieciséis trabajos ${ }^{3}$ realizados por mujeres que se especializaban en diferentes campos, pero que reunían una característica: pensaban el espacio desde una perspectiva de género. En el siguiente cuadro (Cuadro 1) se muestran los títulos de los trabajos presentados, sus autoras/es y procedencia:

A casi 20 años de las Jornadas se presenta una entrevista realizada a Mónica Colombara, en la que se puede identificar su sostenida motivación para instalar la perspectiva de género en los debates geográficos, a pesar de las críticas recibidas por los colegas, incluso en encuentros que se pretendían críticos. Como puede leerse en la entrevista, Mónica Colombara planteó debates acerca de la división sexual del trabajo y el modo de garantizar la sostenibilidad de la vida en esos encuentros de geógrafos, dialo-

2 Puede consultarse la edición completa del número en: http://www.biblioteca.unlpam.edu.ar/ publicaciones/pub-huellas.htm (consultada en agosto de 2018).

3 Los trabajos presentados y el afiche de difusión de las Jornadas pueden consultarse en: https:// geografiadegeneroargentina.wordpress.com/i-jornadas-unlz-1999/ (consultada en agosto de 2018). 
Cuadro 1: Trabajos presentados en las Primeras Jornadas Latinoamericanas de Género y Geografía en la Universidad de Lomas de Zamora.

\begin{tabular}{|c|c|c|c|}
\hline Título del trabajo & Autoras y profesión & $\begin{array}{l}\text { Pertenencia } \\
\text { institucional }\end{array}$ & Lugar de procedencia \\
\hline $\begin{array}{l}\text { Claroscuros y movimientos en } \\
\text { torno a nuestra subjetividad. }\end{array}$ & $\begin{array}{l}\text { Guillermina Berkunsky Idiart } \\
\text { (Psiquiatra y psicoanalista) }\end{array}$ & $\begin{array}{l}\text { GMSM / APCPBA/ } \\
\text { APA }\end{array}$ & $\begin{array}{l}\text { Tandil, Prov de Bs } \\
\text { As, Arg. }\end{array}$ \\
\hline $\begin{array}{l}\text { Género y ciudadanía en la institu- } \\
\text { ción escolar. }\end{array}$ & $\begin{array}{l}\text { Mónica Colombara, et al } \\
\text { (Geógrafa y estudiantes de } \\
\text { Geografía) }\end{array}$ & ISFD N ${ }^{\circ} 41$ & $\begin{array}{l}\text { Alte Brown, Bs As, } \\
\text { Argentina }\end{array}$ \\
\hline $\begin{array}{l}\text { Estructura y desigualdad de opor- } \\
\text { tunidades en la UNSa. }\end{array}$ & $\begin{array}{l}\text { Alicia R. Dib (Especialista en } \\
\text { Antropología) }\end{array}$ & $\begin{array}{l}\text { Consejo de Investi- } \\
\text { gación, UNSa }\end{array}$ & Salta, Argentina \\
\hline $\begin{array}{l}\text { Más allá de la doble jornada. El } \\
\text { caso de las jefas de hogar en Toay, } \\
\text { Provincia de La Pampa. }\end{array}$ & Leticia García (Geógrafa) & UNLPam & La Pampa, Argentina \\
\hline Género, tiempo y espacio urbano. & Lourdes Gómez Beato & UPO & Sevilla, España \\
\hline $\begin{array}{l}\text { Percepción de mujeres de clase } \\
\text { baja y media en los cambios en los } \\
\text { roles familiares. }\end{array}$ & Lucía E. Griselli (Socióloga) & UBA & CABA, Argentina \\
\hline $\begin{array}{l}\text { La mujer y la representación } \\
\text { parlamentaria. }\end{array}$ & Beatriz Grosso & Sin datos & Argentina \\
\hline $\begin{array}{l}\text { Procesos globales e implicancias } \\
\text { locales en el uso del tiempo y del } \\
\text { espacio, según género, en Tandil. }\end{array}$ & $\begin{array}{l}\text { Diana Lan, Sandra Gómez, } \\
\text { Josefina Di Nucci y Claudia } \\
\text { Mikkelsen (Geógrafas) }\end{array}$ & $\begin{array}{l}\text { Centro de Investiga- } \\
\text { ciones Geográficas } \\
(\mathrm{CIG}), \text { UNCPB }\end{array}$ & $\begin{array}{l}\text { Tandil, Provincia de Bs } \\
\text { As, Argentina }\end{array}$ \\
\hline Memoria y educación. & $\begin{array}{l}\text { Yolanda Lima Lobo (Dra. en } \\
\text { Educación) }\end{array}$ & UFRJ & Rio de Janeiro, Brasil \\
\hline $\begin{array}{l}\text { La espacialidad de la produc- } \\
\text { ción- reproducción en la esfera } \\
\text { doméstica. }\end{array}$ & Silvia López Estrada & $\begin{array}{l}\text { Colegio de la Fron- } \\
\text { tera Norte }\end{array}$ & Tijuana, México \\
\hline $\begin{array}{l}\text { Género, derechos y ciudadanía. } \\
\text { Mujer y participación ciudadana. }\end{array}$ & Marta Loyola & Sin datos & Sin datos \\
\hline $\begin{array}{l}\text { Mujeres y cultura: el (re)conoci- } \\
\text { miento desde la diferencia y el en- } \\
\text { cuentro en la complementariedad. }\end{array}$ & Liliana Medina y Mónica Rojas & UNSa & Salta, Argentina \\
\hline $\begin{array}{l}\text { ¿Cuál desarrollo humano para las } \\
\text { mujeres? }\end{array}$ & María Julia Palacios & UNSa & Salta, Argentina \\
\hline $\begin{array}{l}\text { Geografía y género: mujer, familia } \\
\text { y trabajo. Ribeirao Preto y su } \\
\text { entorno, San Pablo, Brasil. }\end{array}$ & Rosa Ester Rossini (Geógrafa) & $\begin{array}{l}\text { Departamento de } \\
\text { Geografia, USP }\end{array}$ & San Pablo, Brasil \\
\hline $\begin{array}{l}\text { Identidad y subjetividad femenina } \\
\text { al final del milenio. }\end{array}$ & $\begin{array}{l}\text { Carmen Secades y Noemi } \\
\text { Ciarlantini }\end{array}$ & AASES & CABA, Argentina \\
\hline $\begin{array}{l}\text { Mujeres y movimientos de acción } \\
\text { ambiental. }\end{array}$ & Nidia S. Tadeo (Geógrafa) & $\begin{array}{l}\text { Geografía de la } \\
\text { UNLP }\end{array}$ & $\begin{array}{l}\text { La Plata, Prov de Bs } \\
\text { As, Arg. }\end{array}$ \\
\hline $\begin{array}{l}\text { Prácticas anticonceptivas. La opción } \\
\text { por la esterilización quirúrgica en el } \\
\text { municipio de Río Grande/RS/Brasil. }\end{array}$ & $\begin{array}{l}\text { Susana Veleda Maria da Silva } \\
\text { (Geógrafa) }\end{array}$ & UFRJ & Rio de Janeiro, Brasil \\
\hline
\end{tabular}

Fuente: elaboración propia.

gando con las temáticas que se venían desarrollando desde otras disciplinas (como la Economía o la Sociología). En la entrevista también se pone en juego un modo feminista de construir conocimiento, esto es, la formación a partir del debate con otras/os, la posibilidad de participación sin requisitos excluyentes, el diálogo con otros saberes validados por la praxis política o por disciplinas cercanas a la Geografía y el continuo cuestionamiento y reflexión sobre las categorías de análisis utilizadas para comprender y explicar la realidad. 
A su vez, en la entrevista se evidencia la necesidad de generar redes entre aquellas geógrafas que investigaban las cuestiones de género y/o desarrollaban una práctica política feminista, independientemente del lugar del mundo en el que se desarrollasen. Esta necesidad se basaba en la falta histórica de producción geográfica con perspectiva de género. Las redes tendidas en 1999 continúan fortaleciéndose en la actualidad como estrategia de fortalecimiento de la investigación en esta línea de la Geografía. Como puede verse en la figura $\mathrm{N}^{\circ} 1$, Mónica Colombara menciona la participación en las Jornadas de geógrafas de diferentes partes del mundo como luces en la producción de Geografía de Género. De esta manera, se ratifica que estas geógrafas no estaban en soledad, sino que, a pesar de las distancias kilométricas, todas trabajaban para aportar al desarrollo de una Geografía comprometida en evidenciar y transformar las desigualdades de género.

Figura $\mathrm{N}^{\circ}$ 1. Mapa de los lugares de procedencia de las participantes de las Jornadas

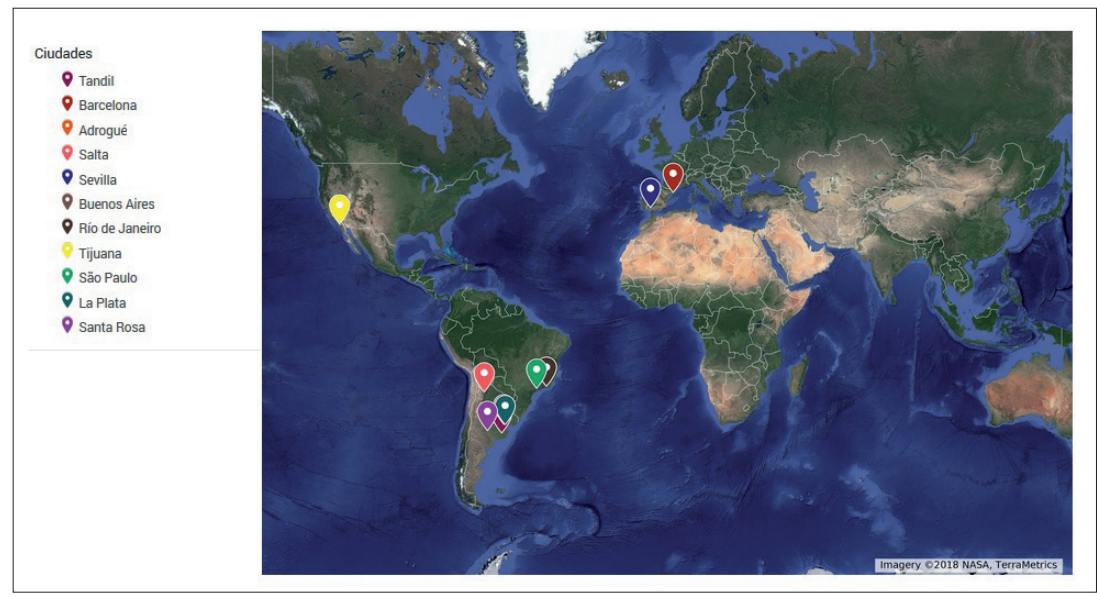

Fuente: elaboración propia en base a Google Maps.

\section{Entrevista a Mónica Colombara}

\section{¿Cómo surgió la idea de organizar unas Jornadas de Geografía y género?}

Sí que ha habido cosas interesantes, mirándolo con el tiempo. Yo en esos años era integrante de la Universidad de $\operatorname{Lomas}^{4}(. .$.$) , dependía direc-$ tamente de Extensión de la Universidad. Y el equipo de gente con el que

4 La UNLZ fue creada el 13 de octubre de 1972 por el decreto 19.888/72. Está ubicada en la Provincia de Buenos Aires en la zona sur del Conurbano bonaerense. Para más información sobre la institución, puede consultarse el sitio web oficial: http://www.unlz.edu.ar (consultado en junio de 2018). 
trabajaba, bueno, me conocía y sabía cuál era mi mirada del tema. (...) Lo cual me dio pie a pedirle a la Universidad que me respaldara en algunos trabajos y algunas ideas que yo tenía. Y le dieron el ok. (...) No fueron muchos días, si mal no recuerdo, fueron dos días enteros. (...) Lo que ocurre es que tampoco teníamos muchos vínculos con otros Centros de Geografía. Ahora que estoy recordando, yo para ese entonces había hecho un trabajo, también desde la Universidad, un relevamiento de cuál era la composición de los Institutos y Departamentos de Geografía de las Universidades Nacionales del país. Porque yo me basaba un poco en lo que habían hecho las inglesas ${ }^{5}$, que después fue copiado por las españolas ${ }^{6}$ : qué pasaba en la Geografía académica dentro de las universidades, cómo estaba conformado a nivel de alumnos, ayudantes de cátedra, profesores, editoriales de las revistas, etc. Entonces yo lo hice para la Argentina, lo cual, si no hubiera tenido el apoyo explícito de la Universidad, con el aval y con la presentación, me hubiera costado bastante. No obstante, todas las universidades me contestaron en lo largo de un año. Yo hubiera preferido que hubiera sido tres meses, pero fue a lo largo de un año. Pero pude hacer algo y salió interesante. (...) Otro tema que me pareció: cómo estábamos en la Geografía de Género en la Argentina. $Y$ en esto me parece que esos años previos, esto fue en el '99, desde el '91/'93, es cuando empiezan a aparecer algunos artículos anclados, por lo menos, presentado en alguna actividad académica, podía ser una jornada, un congreso, etc. Pero a nivel personal fue muy interesante haber hecho contacto con gente de Latinoamérica a raíz de haber ido a los Encuentros de Geógrafos de América Latina (EGAL) ${ }^{7}$. Yo empiezo a ir en el segundo en 1989 en Uruguay $^{8}$ y ahí, si bien recibí muchas cargadas, muchas "burlas" machistas, inclusive alguna interpelación fuerte exponiendo el trabajo sobre el tema de las masculinidades. Los que más reaccionaron eran los hombres.

5 Mónica Colombara hace referencia al trabajo de Linda Mcdowell publicado en 1979 que se titula "Women in British geography" ("La mujer en la Geografía británica").

6 La entrevistada se refiere a los siguientes artículos escritos por las geógrafas pertenecientes al grupo de investigación de Geografía y género de la Universitat Autònoma de Barcelona (UAB): "La mujer y la geografía universitaria española" de Margarida Castañer i Vivas y Núria Centelles i Rabadan; "El papel de la mujer en el desarrollo de la geografía." de García Ballesteros, publicado en el libro Liberación y Utopía en 1982; y "Mujer, Geografía y feminismo" de Ana Sabaté Martínez, publicado en Anales de Geografía de la Universidad Complutense de Madrid en 1984.

7 Los Encuentros de Geógrafos de América Latina (EGAL) se realizan desde el año 1987, con una periodicidad de 2 años en diferentes países de América Latina.

8 El II EGAL se realizó en la ciudad de Montevideo, Uruguay, del 27 al 31 marzo de 1989 y contó con la participación de Geógrafos/as de Argentina, Brasil, Costa Rica, Cuba, Chile, Ecuador, México, Panamá, Venezuela, España y Uruguay. 


\section{¿Cuáles fueron esas críticas?}

Me acuerdo que era un trabajo sobre los roles de género y estaba vinculado con la espacialidad: que el rol de las mujeres es ocuparnos muchas veces de nuestros hijos, de llevarlos a las escuelas, que además de los trabajos de cuidado teníamos otros trabajos como fuera el trabajo formal, etc. Y que eso insumía tiempo en las mujeres para pensar en sí mismas o para hacer trabajos que tenían que ver justamente con irrumpir en el espacio público. Y me acuerdo que un venezolano desde el fondo me dijo que él estaba totalmente en desacuerdo. ¿Y por qué? Porque lo personalizó. Porque dijo "yo soy el que en mi casa lleva todos los días a la escuela a mis hijos y soy el que hago las compras" y no sé qué otra cosa más me dijo, enojado. Entonces yo le contesté que estos eran temas de índole cultural y que había muchas conductas que las teníamos totalmente naturalizadas e invisibilizadas y que de ninguna manera era un ataque hacia su persona, que él no podía desestimar ni desconocer que quizás él era la excepción. (Risas.) (...) Y volviendo a lo anterior, yo tenía entonces algunos contactos, creo que a través de las lecturas del EGAL empecé a recibir contactos de personas que estaban pensando cosas parecidas. Y es así que nos encontramos en un momento determinado con Leticia García ${ }^{9}$, fue muy interesante. Ya estaba Diana Lan $^{10}$. Ya como empezar a encontrarte en estos encuentros con intereses comunes en el '91. En el '89 fui a ver de qué se trataba, por más de que ya tenía conciencia de género, fui a ver de qué se trataba. Y ahí, llamativamente, una Geografía que se propone radical, por qué no se trataban y aún no se tratan estos temas. Porque tan radical me parece que no es, o dejó de serlo. No recuerdo bien, pero me parece que fue en el EGAL de Chile ${ }^{11}$, fue en 2001 donde formamos una red. Porque este seminario sirvió para armar la primera red, pero no teníamos celulares, algunas no teníamos ni siquiera correo electrónico. Entonces también hay que pensar también en eso: las dificultades que se tenían hace 20 años, ¿cómo hacías

9 Leticia García, una de las primeras geógrafas en incorporar la mirada de género, es Especialista en Estudios Sociales y Culturales de la UNLPam desde 2012. Dicta el Seminario de Investigación Geográfica IV (con especialidad en Geografía de Género) de la Licenciatura en Geografía de la misma universidad. Integra la Cátedra extracurricular de Educación Sexual Integral, derechos sexuales y reproductivos de la UNLPam y la Comisión de Intervención institucional ante situaciones de violencia de género, acoso sexual y discriminación de género en UNLPam.

10 Diana Lan es una de las geógrafas que viene desarrollando de manera sostenida la perspectiva de género en la Geografía. Es Directora del Centro de Investigaciones Geográficas. IGEHCSCONICET/UNCPBA y Doctora en Geografía por la UNLP. Es co-directora de la "Revista Latinoamericana de Geografía y género" desde 2009. Escribió numerosos artículos y capítulos de libros sobre Geografía de género y los "circuitos espaciales de la violencia".

11 El VIII EGAL se realizó en Santiago de Chile entre el 4 y el 10 de marzo de 2011. 
para estar comunicada? Había que buscar muchas estrategias. ¿A quién invitamos? Invitamos a Rosa Rossini ${ }^{12}$, que la había conocido en los EGAL, sobre todo, el de San $\mathrm{Pablo}^{13}$, y algún otro encuentro más al que fui. (...) Y entonces ¿qué hacía? Yo buscaba bibliografía, charlaba con uno y con otro, haces contacto. $\mathrm{Y}$ esos contactos nos permitieron invitarlas. (...) Las que vinieron no tienen un perfil fuertemente geográfico. Pero les llamó mucho la atención de una Geografía de Género a gente que estaba en otras áreas trabajando género. (...) También vino Susana Veleda ${ }^{14}$, yo la conozco ahí. Yo no la conocía de antes. (...). Después vino, de afuera, Rosa Rossini y Susana Veleda. Y vino López Estrada de México. Y de España. ¡Hace 20 años! Vinieron una de España, dos de Brasil y una de México. Ahora me doy cuenta, hace 20 años ya había como lucecitas prendiéndose en estos lugares. $\mathrm{Y}$ ahí conformamos una primera red para pasarnos información, o algún trabajo que pasara o si sabíamos de algún encuentro. (...)

\section{¿La modalidad de las Jornadas se concretó como fue anunciada, es decir, en conferencias y mesas redondas de discusión?}

Fue una mesa redonda más que una conferencia. Una mesa redonda donde Rosa Rossini exponía, porque era la más pope en ese momento porque tiene una mirada comprometida de género. (...)

\section{¿Cuáles eran los requisitos para presentar una ponencia y poder par- ticipar en las Jornadas?}

Ninguno. Cualquiera que le interesara el tema. Un poco siguiendo la temática de los Encuentros que después se ha desvirtuado bastante. Porque en los EGAL el espíritu inicial era ese: encuentro. La palabra es esa, no es ni congreso, ni jornada, ni seminario, ni nada de eso, encuentro. (...)

\section{¿Cómo fue la dinámica de trabajos en esos días de las Jornadas?}

Todos escuchan a todos. Se exponían, se hacían preguntas, se discutía y como, eran 20 trabajos, daba para dos días, con una mesa redonda. Los trabajos me los mandaban previamente a mí por correo, los leía y no veté

12 Rosa Rossini fue una de las primeras geógrafas brasileñas en trabajar la cuestión de género. Profesora del Departamento de Geografía de la USP desde 1971, Doctora en Geografía Humana (1975). Fue homenajeada por CNPq en Pioneiras de la Ciencia de Brasil - $4^{\mathrm{a}}$ Edición en octubre de 2014.

13 En 2005 se llevó a cabo el X EGAL en San Pablo, Brasil. Llevó por lema "Por una geografía latinoamericana: del laberinto de la soledad, al espacio de la solidaridad".

14 Susana María Veleda Da Silva es Doctora en Geografía Humana por la UAB. Es Investigadora del Grupo de Investigación de Geografía y Género de la UAB, representante de América Latina en la Comisión de Geografía y Género de la UGI en el período 2008-2012. 
ninguno, porque no me interesaba, al contrario, la idea era incluirlo. Inclusive había un aprendizaje porque sobre eso también se hacían evaluaciones verbales (...) Para mí fue bien horizontal, no se le dio una estructura jerárquica, de académico estricto (...) Yo recibía el trabajo y me ponía contenta de recibir el trabajo. Organicé por temáticas los trabajos. (...)

\section{¿Cuáles fueron las principales ideas o debates que circularon en las Jornadas?}

Había propuestas más que nada sobre los temas de los trabajos. Y el tema de lo teórico como en construcción, porque eran pocos los que tenían un marco teórico armado. (...) En algunos casos sí, más que en otros, obviamente. Aquellos que están acostumbrados a mandar a la academia y mandan trabajos, tienen como otra lógica. Pero también había gente de ONG (...) Era mucho más transversal, horizontal. Era hasta interdisciplinario porque salió mucho el tema del feminismo, de la acción feminista, más que las cuestiones teóricas como estaríamos planteando hoy.

¿Existieron debates centrados en la especificidad geográfica? ¿Las discusiones incorporaban de alguna manera los conceptos geográficos?

No, eran más feministas. No eran debates geográficos del espacio o del lugar. Estaba siempre el espacio.

Quienes participaron no tenían necesariamente un bagaje teórico desde la Geografía, ¿pero las categorías que se pusieron en discusión tanto a nivel teórico como práctico en las Jornadas eran geográficas?

Sí, las categorías eran geográficas y nadie las discutía porque consideraban que para todos eran lo mismo (...). No había una discusión sobre las categorías geográficas, había una apropiación sin discutir porque era útil a otras demandas.

¿Y las geógrafas que participaron de las Jornadas estaban más interesadas en dar debates sobre las categorías conceptuales de la Geografía o de la Teoría de Género?

La importancia no estaba en las categorías conceptuales de geografía, sino en las de género. (...) Lo ideal sería cómo se pueden aunar las dos cosas: yo estoy hablando de feminismo pero también estoy hablando de lugar, estoy hablando de territorio. No sé, tal vez el desafío futuro es ese.

Mirándolo a la distancia temporal, ¿qué impresiones tiene sobre usted misma a casi 20 años de la organización y realización de las Jornadas? 
Como una locura. Me veo con la audacia y el desafío de alguien que tiene la energía para hacerlo y que no le importan los obstáculos que pueda tener porque estaba convencida de que había que hacerlo. Y además darle un mayor empuje a estas cuestiones. (...) También mi mirada desde la militancia feminista y también como partícipe de una organización que trabaja temas de violencia desde hace 30 años a mí me dio una mirada comprometida, pero aparte, me dio una mirada de que todo es posible. (...) Pero esa red que se formó ahí muy embrionaria se mantuvo, porque hay varias de las que estábamos en esa red, que hoy seguimos conectadas: Susana Veleda, Rosa Rossini, Diana Lan, Leticia García. Se hizo. (...) Me veo muy audaz. 20 años menos, es mucho. A esta altura, 20 años menos, es mucho. Y además en una edad... eso también es interesante... yo he leído una presentación que hizo Janice Monk en un congreso pero hace un paralelo entre su vida personal y su vida académica. Es como un curriculum autorreferenciado. Y dije "qué bueno lo que está diciendo esta mujer". Porque uno es en su tiempo, es en su espacio, en Buenos Aires, Argentina, en los años '90. (...)

\section{Así como fue una gran influencia en cuestiones de género para muchas geógrafas, ¿quién fue su Mónica Colombara? ¿De quién aprendió a incorporar la mirada feminista?}

La mirada feminista a mí me la dio la Fundación ${ }^{15}$, que por algo la formamos. (...) la mayoría eran psicólogas sociales y ellas fueron al frente de batalla. Y una de ellas un día me cuenta que salió un artículo muy interesante sobre una arquitecta que diseña casas con las mujeres de acuerdo a las necesidades de las mujeres, que se llamaba Teresa Azcárate ${ }^{16}$. Resultó que era pariente mía y fue con la primera que me puse a hablar de espacios y mujeres porque ella estaba muy interesada en espacios acotados. (...) Teresa Azcárate fue mi inspiradora (...).

A casi 20 años, si tuviera la posibilidad de volver a organizar las Jornadas, ¿repetiría la lógica de organización y la modalidad de trabajo? ¿Qué cambios haría? ¿Qué balance general sobre las Jornadas puede hacer?

(Silencio). Si las llamo de nuevo Jornadas, pasaría a llamarlas Jornadas de Geografía Feminista e invitaría no sólo a las académicas sino a gente

15 La Fundación Propuesta, localizada en la zona sur del Gran Buenos Aires, fue fundada en 1989. Está conformada por un equipo interdisciplinario que trabaja sobre situaciones de violencia de género.

16 Teresa Azcárate es Arquitecta y argentina. Es especialista en temas de mujer, hábitat y medio ambiente. Pertenece a la Asociación de Especialistas Universitarios en Estudios de la Mujer (ADEUEM). 
que está trabajando en los territorios no sólo con cuestiones de género sino también de sexualidades. Me parece que el espectro ha cambiado. Y tal vez haría un panel para poner en discusión las categorías propias de la Geografía, pero sin olvidarme nunca de lo que pasa en el territorio. También organizaría un espacio... como los trabajos seguramente no van a ser muchos, a mí me parece que todas tienen que escuchar a todas. Quizás lleva un poco más de tiempo, pero no dividir por temáticas. Y después también pondría algo así como un conversatorio o una buena entrevistadora a alguien destacado (...) y que despliegue todo lo que nos pueda dar de su formación.

\section{¿Por qué pasaría de llamarlas "Jornadas de Geografía de Género" a "Jornadas de Geografía feminista"?}

Por el mayor compromiso político, por la mayor militancia, para interpelar un poco a la academia. También podría ser "Geografía de las sexualidades", ambas. Por el carácter más político, más por eso. Hoy digo, si nos movemos en el ámbito académico pienso que tendríamos que decir "Geografía de género" (...) yo me animaría a decir que en el futuro vamos a hablar de sexualidades, pero no sé si está todo el mundo preparado para ya hablar de sexualidades. Pero para mí hay que hablar de sexualidades.

\section{Referencias bibliográficas}

CASTEÑERI VIVAS, M. y CENTELLES I RABADAN, N. (1985). La mujer y la geografía universitaria española, Documents d'Anàlisi Geográfica $\mathrm{N}^{\circ}$ 7, pp. 103-140. Disponible en: https://www.raco.cat/index.php/DocumentsAnalisi/article/viewFile/41367/52200

DILLON, B. (1999). "La mitad del cielo". Una introducción a la geografía del género, Revista Huellas, Número especial, pp. 11-13. Disponible en: http://www.biblioteca.unlpam.edu. ar/pubpdf/huellas/Espa02Dillon.pdf

GARCÍA BALLESTEROS, A. (1982). El papel de la mujer en el desarrollo de la Geografía. En M. A. DURAN (Ed.), Liberación y Utopía (pp. 119-141). Madrid, España: Akal Universitaria.

GEOGRAFIA DE GENERO DE ARGENTINA (2017). https://geografiadegeneroargentina. wordpress.com

MCDOWELL, L. (1979). Women in British Geography. Area, vol. 11, núm. 2, pp. 151-154.
SABATÉ MARTÍNEZ, A. (1984). Mujer, Geografía y feminismo, Anales de Geografia de la Universidad Complutense de Madrid, vol. IV, pp. 37-56. Disponible en: https://revistas.ucm.es/index.php/AGUC/article/view/ AGUC8484110037A/3211

UNIVERSIDAD DE LOMAS DE ZAMORA (s/f) http://www.unlz.edu.ar 